\title{
USING THE PATH CODE FOR MODELING GRADUAL SEP EVENTS IN THE INNER HELIOSPHERE
}

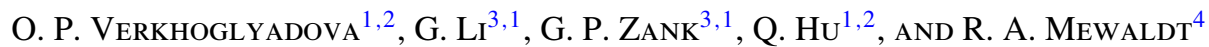 \\ ${ }^{1}$ Center for Space Plasma and Aeronomy Research, University of Alabama in Huntsville, Huntsville, AL 35899, USA \\ 2 IGPP, University of California, Riverside, CA 92521, USA \\ ${ }^{3}$ Department of Physics, University of Alabama in Huntsville, Huntsville, AL 35899, USA \\ ${ }^{4}$ California Institute of Technology, Pasadena, CA 91125, USA \\ Received 2007 October 8; accepted 2008 November 21; published 2009 March 3
}

\begin{abstract}
We model a gradual solar energetic particle (SEP) event that occurred on 2001 September 29, and was possibly caused by a coronal mass ejection related shock. A computer code PATH (particle acceleration and transport in the heliosphere) was tuned to simulate this event. The model includes local particle injection at an evolving quasiparallel shock, first-order Fermi acceleration at the shock, and self-consistent excitation of MHD waves to enhance particle scattering, particle trapping, and escape from the shock complex, and transport in the inner heliosphere up to several AU. The shock and solar wind boundary conditions are derived from Advanced Composition Explorer $(A C E)$ observations at $1 \mathrm{AU}$, which are then extrapolated to $0.1 \mathrm{AU}$. Modeled time-dependent spectra for energetic protons, iron, and oxygen ions are compared with ULEIS and SIS measurements onboard $A C E$, and with $G O E S-8$ data. The use of the PATH code to model gradual SEP events superimposed on a pre-event background from previous SEPs is discussed.
\end{abstract}

Key words: acceleration of particles - MHD - shock waves

Online-only material: color figures

\section{INTRODUCTION}

Gradual solar energetic particle (SEP) events associated with coronal mass ejections (CMEs) can cause a substantial increase in energetic particle fluxes at the Earth's orbit (for example, see Kahler et al. 1984; Kallenrode 2003). These events are manifestations of extreme energy releases at the Sun and pose radiation hazards at near-Earth orbits. Accelerated particles in these events can reach energies up to several $\mathrm{GeV}$, directly affecting spacecraft in the inner heliosphere. A CME travels 1 AU within about 2 days on average and particles are continuously injected into its shock complex. For this reason, it is important to understand how these particles are accelerated at an evolving shock and make realistic predictions of the temporal evolution of energetic particle fluxes and spectra at 1 AU.

The following study focuses on modeling a specific SEP event and associated ESP (energetic storm particle) event observed on UT 2001 September 29, at 9:06. This particular event occurred during a series of large gradual SEP events that started on September 24 and ended on September 30 (see Event\# 25 in Tylka et al. 2005; Event\# 33 in Desai et al. 2006; and a review by Gopalswamy et al. 2003). The SEP event of September 29 was selected from the Advanced Composition Explorer ( $A C E$ ) interplanetary shock databases (see below for details) by the following selection criteria: (1) we require the event to be associated with a quasi-parallel shock observed at 1 AU by $A C E$ and (2) we require that a local ESP phase is observed in the SEP event. The first requirement is mainly a technical one, as we will be using a one-dimensional code that is based on a quasi-parallel shock configuration; thus, a potential good candidate is a shock that maintains this configuration throughout its propagation. Since we know little about the angle between a shock normal and the background magnetic field when the shock is close to the Sun, we start by requiring candidate events to have a local quasi-parallel shock configuration at $1 \mathrm{AU}$. The second requirement of a local
ESP phase ensures that particle acceleration is occurring at the shock. Indeed, many shocks (even fast ones) do not accelerate particles. The reasons could be a lack of seed population and/ or low injection efficiency. We note that the shock we study in this paper, the 2001 September 29 shock, occurred in the wake of a much stronger shock of September 24 (Tylka et al. 2005). The presence of an earlier stronger shock can enhance the interplanetary turbulence level and increase the seed population. We will show that particles accelerated at the 2001 September 29 shock are clearly observed in iron and oxygen ion fluxes below or about $1 \mathrm{MeV}$ at least $\sim 30 \mathrm{hr}$ prior to this shock arrival (see Section 4).

Recently, several approaches to model particle acceleration at quasi-parallel shocks in SEP events have been developed (see reviews by Malkov \& Drury 2001; Lee 2005). The numerical model by $\mathrm{Ng}$ (2007) describes the interaction of energetic protons with Alfvén waves and considers wave evolution through wave excitation/damping. This promising theoretical approach yields important results on the evolution of the proton spectrum, proton mean-free path, and wave power spectrum in the shock vicinity. However, the $\mathrm{Ng}$ model does not consider the evolution of the shock as it propagates throughout the heliosphere. The numerical approach presented by Vainio \& Laitinen (2007) addresses time dependence in particle acceleration and Alfvén wave generation at a quasi-parallel shock. Their model results show that these processes can be described by quasi-stationarystate models of shock acceleration, but a more accurate estimate of the maximum achievable energy is required. The preliminary Vainio \& Laitinen (2007) model was tested for a fixed set of solar wind parameters and the shock was treated as a boundary condition (without a description of the downstream region). A comprehensive analytical quasi-linear model by Lee (2005) extends an approach developed in earlier papers (Lee 1983; Gordon et al. 1999). The theory incorporates diffusive shock acceleration (DSA), ion advection, and diffusion in the solar wind, magnetic focusing, and self-consistent wave excitation by 
energetic protons. It describes correctly the main features of the proton spectrum observed in gradual SEP events. However, further effort is needed to describe the observed heavy ion spectra. An interesting approach based on conservation laws and resonance conditions for wave-particle interactions was proposed by Galinsky \& Schevchenko (2007). Their alternative to the DSA mechanism includes a continuous description of the particle distribution without division into a seed population and energetic particles. Thus, the problems of particle injection into the shock and escape are naturally resolved in this model. Pitchangle averaging is not used either. The approach of Galinsky \& Schevchenko (2007) includes shock modification by the accelerated particles (see Malkov \& Drury 2001, for details) and addresses shock stability. Waves are generated self-consistently from the unstable particle distribution.

The code we use here is called PATH (particle acceleration and transport in the heliosphere). A short description of the numerical approach is given in Section 2. Section 3 describes details of the modeling and outlines the main results. We trace a shock throughout the inner heliosphere, and calculate particle fluxes and spectra at $1 \mathrm{AU}$ up to the shockarrival time. A comparison of the modeled spectra with particle detector measurements at $A C E$ and GOES- 8 shows a remarkable agreement. Discussion and conclusions are presented in Section 4.

\section{MODELING PARTICLE ACCELERATION AND TRANSPORT IN THE HELIOSPHERE USING PATH}

The PATH model consists of two major parts; the first part models an evolving shock and particle acceleration, and the second part follows energetic particle transport throughout the heliosphere (from 0.1 to $>1 \mathrm{AU}$ ). The core of the PATH model was introduced in a number of papers (Zank et al. 2000; Rice et al. 2003; Li et al. 2003, 2005), and is briefly described here. Shock propagation and evolution are modeled using a modified ZEUS code developed for astrophysical MHD shock simulations. In this paper, we restrict ourselves to onedimensional modeling. We consider a spherically symmetric shock and assume that all physical parameters depend on radial distance only. This is a reasonable approximation for a quasiparallel shock. The MHD part of the code can be easily modified for two- and three-dimensional cases (Rice \& Zank 2003).

The new feature of the PATH model is its ability to adjust the background solar wind parameters to model the values observed at $1 \mathrm{AU}$ for a particular event. Thus, we expect to reproduce specific dynamics of selected SEP events. This feature is achieved by setting the solar wind density, velocity, magnetic field, and temperature at $0.1 \mathrm{AU}$ through extrapolation of these values observed at $1 \mathrm{AU}$. The MHD code allows us to create a numerical model of the stationary solar wind throughout the simulation domain. We then model a propagating shock through this "background," following the shock-compression ratio and velocity, as they change with radial distance.

Solar wind suprathermal particles (or some pre-existing seed particles) are injected into the shock (the outermost shell) at a certain injection energy which is assumed to be the same for all particle species (Zank et al. 2000). We assume that the injection mechanism at a quasi-parallel shock does not distinguish protons from heavy ions. We follow a self-consistent approach developed in the paper by Zank et al. (1993). The total injected particles and the injection energy are parameters that can be adjusted. We currently take $\sim 10 \mathrm{keV}$ as the injection energy and assume a $1 \%$ of the particle flux density as the injection efficiency (Zank et al. 2000; Li et al. 2005). The DSA process for energetic particles (protons and heavy ions) is then followed at the evolving shock.

Immediately upstream of the shock, enhanced levels of magnetic turbulence for the DSA mechanism are required. We suppose that for a quasi-parallel shock, this enhancement is due to excitation of the upstream Alfvén waves through waveparticle interaction with streaming protons. A self-consistent treatment of this interaction yields a value of the parallel diffusion coefficient (Lee 1983; Gordon et al. 1999; Rice et al. 2003). By scattering on the MHD turbulence, suprathermal particles return to and cross the shock repeatedly, gaining energy in each cycle. This model can be easily extended to include a quasi-perpendicular shock (Zank et al. 2006). The local accelerated particle spectrum at the shock is assumed to be a power-law spectrum corresponding to the instantaneous Mach number and compression ratio $(s)$, which change with heliocentric distance as the shock evolves and background magnetic field weakens. We adopt the following solution for particle distribution function for a planar shock (Zank et al. 2000):

$$
f \sim\left(p / p_{\text {inj }}\right)^{-\beta\left(t_{i}\right)}\left[H\left(p-p_{\text {inj }}\left(t_{i}\right)\right)-H\left(p-p_{\max }\left(t_{i}\right)\right)\right],
$$

where, $H$ is Heaviside step function, $p$ is the particle momentum, $\beta=3 s /(s-1)$ and $s$ is the shock compression ratio. The minimum momentum (energy) for particles is the injection

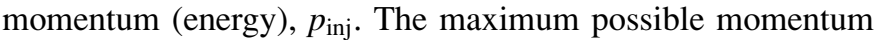
(energy), $p_{\max }$, achieved in the acceleration process at a quasiparallel shock is computed by equating the dynamical time scale of the shock to the acceleration time scale (Zank et al. 2000). This maximum achievable momentum (energy) depends on age and strength of the shock, $\beta$, Mach number, and the interplanetary magnetic field at the instantaneous shock position at $t_{i}$. Heavy ions are treated in a similar manner as the protons, but are assumed to behave as test particles and do not generate waves. The maximum energy is inversely proportional to $(A / Q)^{2}$ and decreases with time as the shock decelerates $(\mathrm{Li}$ et al. 2005). Thus, our model imposes the instantaneous powerlaw spectra (Equation 1) at the shock up to the maximum energy achievable locally. Particle spectra above that energy are formed due to transport to $1 \mathrm{AU}$ and can develop into exponential rollover or double-power-law spectra.

The PATH model solves the kinetic transport equation for energetic particles in the immediate vicinity of the shock, using the isotropic diffusive approximation and an operatorsplitting approach. This approach admits an attractive physical interpretation in terms of nested shells of solar wind material (Zank et al. 2000). By means of the shell approach, we can follow particle acceleration, diffusion, and cooling in terms of shells. Particles convect with a shell and diffuse to other shells. An energetic particle accelerated at the shock no longer propagates diffusively once it reaches an escape distance ahead of the shock. At this point, we suppose that the particle has escaped into the interplanetary medium and the subsequent transport is treated separately.

Particle transport outside the shock complex is described in the second part of the PATH model using the Monte Carlo approach of $\mathrm{Li}$ et al. $(2003,2005)$. The mean-free path $\lambda$ is a function of particle momentum $p$ and heliocentric distance $r$

$$
\lambda=\lambda_{0}\left(\frac{p c}{1 \mathrm{GeV}}\right)^{\alpha} \cdot\left(\frac{r}{1 \mathrm{AU}}\right)^{\beta},
$$

where parameters are chosen to be $\lambda_{0}=0.8 \mathrm{AU}, \alpha=1 / 3$, 
$\beta=2 / 3$ in this study. Particles that escape from the shock complex follow interplanetary magnetic field lines while experiencing scattering in pitch angle. Besides scattering, a particle also experiences magnetic focusing and adiabatic cooling. We do not consider field line meandering in the model. The output of the code is dynamical spectra, including particle anisotropies, and fluxes of protons and heavy ions in arbitrary units (see below) at $1 \mathrm{AU}$, which can be compared with in situ observations.

\section{MODELING RESULTS}

\subsection{Evolving Shock and Particle Acceleration}

We apply the PATH model to simulate particle spectra and fluxes at $1 \mathrm{AU}$ in the SEP event, which corresponds to the shock of 2001 September 29. We assume that the energetic particles are produced by DSA at the CME-driven shock detected by $A C E$ at UT 9:06. We refer to the $A C E$ List of Disturbances and Transients (maintained by C.W. Smith at http://www-ssg.sr.unh.edu/mag/ace/ACElists/obs_list.html) and MIT shock database (maintained by J. Kasper at http:// space.mit.edu/home/jck/shockdb/shockdb_files/acemain.htm) for the observed shock and solar wind parameters at $1 \mathrm{AU}$. The average angle between the shock normal and the background magnetic field is estimated to be $\sim 20^{\circ}$ (another estimate gives 25.7), which justifies the approximation of a quasi-parallel shock at $1 \mathrm{AU}$. In general, a CME-associated shock does not maintain the same angle to the local magnetic field line during its propagation from the Sun to $1 \mathrm{AU}$. A two-dimensional model is needed to treat properly the evolution of the shock geometry. In our one-dimensional modeling, we assume that the shock remains quasi-parallel. Our results show that the model provides a reasonable approximation for this particular SEP event. To set boundary conditions, we extrapolate the solar wind and shock parameters from $1 \mathrm{AU}$ to $\sim 0.1 \mathrm{AU}$ by assuming the following scalings: the interplanetary magnetic field radial and azimuthal components in the spherical coordinates at $r_{0}=0.1 \mathrm{AU}$ are obtained through $B_{r}\left(r=r_{0}\right)=$ $B_{r}(r=1 \mathrm{AU}) \cdot\left(1 \mathrm{AU} / r_{0}\right)^{2}, B_{\phi}\left(r_{0}\right)=B_{\phi}(1 \mathrm{AU}) \cdot\left(1 \mathrm{AU} / r_{0}\right)$; the proton number density is $n\left(r_{0}\right)=n(1 \mathrm{AU}) \cdot\left(1 \mathrm{AU} / r_{0}\right)^{2}$. The physical parameters are derived from $A C E$ measurements at $r \sim 1$ AU. We choose the following values for the solar wind density, $n=3 \mathrm{~cm}^{-3}$, interplanetary magnetic field components $B_{r}=4 \mathrm{nT}$ and $B_{\phi}=-3.6 \mathrm{nT}$. We note that a simple estimate shows that an angle between the interplanetary magnetic field direction and the anti-Sun direction is $\sim 42^{\circ}$ at the Earth's orbit and $\sim 5^{\circ}$ near the Sun at $0.1 \mathrm{AU}$ if we assume the Parker spiral configuration. An interplanetary CME distorts the spiral structure of the background magnetic field. Thus, the angle between the shock normal and the background magnetic field is not directly defined by the direction of the undisturbed spiral heliospheric magnetic field and is not constant throughout the shock front. We assume the angle of $20 \mathrm{deg}$ at $1 \mathrm{AU}$ which was derived from $A C E$ observations. Since we observe particles at $1 \mathrm{AU}$ which were accelerated at an early shock, we also assume that the field lines we are on were connected to the shock at $0.1 \mathrm{AU}$.

The above scaled parameters were used in the initial set of boundary conditions to construct a numerical model of the stationary solar wind background. The shock was launched at $r_{0}=0.1 \mathrm{AU}$, and a number of iterations were made to adjust the boundary conditions for both the upstream solar wind and the shock until we achieved a reasonably good fit to the observed values at $1 \mathrm{AU}$ (see Table 1). After iterating, we found boundary conditions for the solar wind velocity $V_{\mathrm{sw}}=425 \mathrm{~km} \mathrm{~s}^{-1}$ and

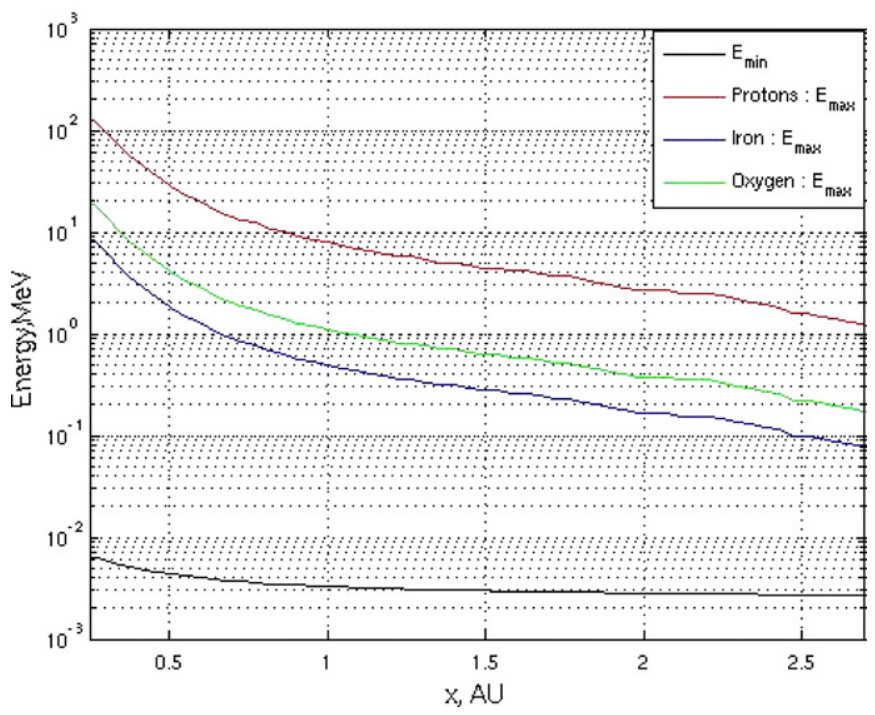

Figure 1. Dynamical evolution of the maximum energies for protons (red), oxygen (green), and iron (blue) ions as the quasi-parallel shock propagates from $\sim 0.1$ AU. The minimum energy (shown in black) is the same for all species.

(A color version of this figure is available in the online journal.)

Table 1

Comparison of the Observed and Modeled Upstream Solar Wind Velocity $\left(V_{\mathrm{sw}}\right)$, Shock Velocity $\left(V_{\mathrm{sh}}\right)$, and Compression Ratio $(s)$

\begin{tabular}{lccc}
\hline \hline Obs./Model & $V_{\text {sw }}, \mathrm{km} \mathrm{s}^{-1}$ & $V_{\text {sh }}, \mathrm{km} \mathrm{s}^{-1}$ & $s$ \\
\hline 1 AU (from ACE data) & $537.4 \pm 4.2$ & $687.6 \pm 34.2$ & $2.31 \pm 0.3$ \\
1 AU (from the PATH model) & 592 & 714 & 2.57 \\
\hline
\end{tabular}

the shock velocity $V_{\mathrm{sh}} \simeq 900 \mathrm{~km} \mathrm{~s}^{-1}$ at $0.1 \mathrm{AU}$. According to our modeling results, the shock reaches $1 \mathrm{AU}$ in $\sim 50 \mathrm{hr}$, allowing us to trace the solar progenitor back to the Sun. There were two partial halo events on September 27, at 04:54:05 and 08:06:05 (online CME catalog, courtesy of CDAW Data Center) associated with flare-produced increases in the X-ray flux measured by GOES-10. Based on our $V_{\text {sh }}$ estimate, each of these events (or possibly both) is a good candidate for the solar cause of the ESP event of September 29 discussed here.

At the next step, we model particle acceleration due to DSA and the transport of protons and heavy ions, namely oxygen ions with a charge to mass ratio of $6: 16$, and iron ions with a ratio of 14:56, in the vicinity of a quasi-parallel shock. As the shock propagates, it slows down and the background magnetic field decreases. Thus, maximum energies to which the particles of different species can be accelerated decrease with increasing heliocentric distance. This effect is illustrated in Figure 1 for different ion species. The ordering of the maximum energies for the different ion species reflects a $(Q / A)^{2}$ dependence as expected for a quasi-parallel shock ( $\mathrm{Li}$ et al. 2005).

\subsection{Particle Transport and Observed Spectra at $1 \mathrm{AU}$}

Accelerated particles eventually escape from the shock and reach $1 \mathrm{AU}$ and beyond. Figure 2 presents modeled spectra for protons, oxygen, and iron ions at $1 \mathrm{AU}$. The spectra were integrated over the time interval of $\sim 24 \mathrm{~h}$ before the modeled shock arrives at $1 \mathrm{AU}$. This time interval was selected to minimize the effects of the preceding powerful shock (see Discussion). A straight line, which corresponds to the theoretical limit of $\sim(s+2) /(s-1) / 2$ as determined by the shockcompression parameter, $s$, at $1 \mathrm{AU}$ (Ellison \& Ramaty 1985), is 

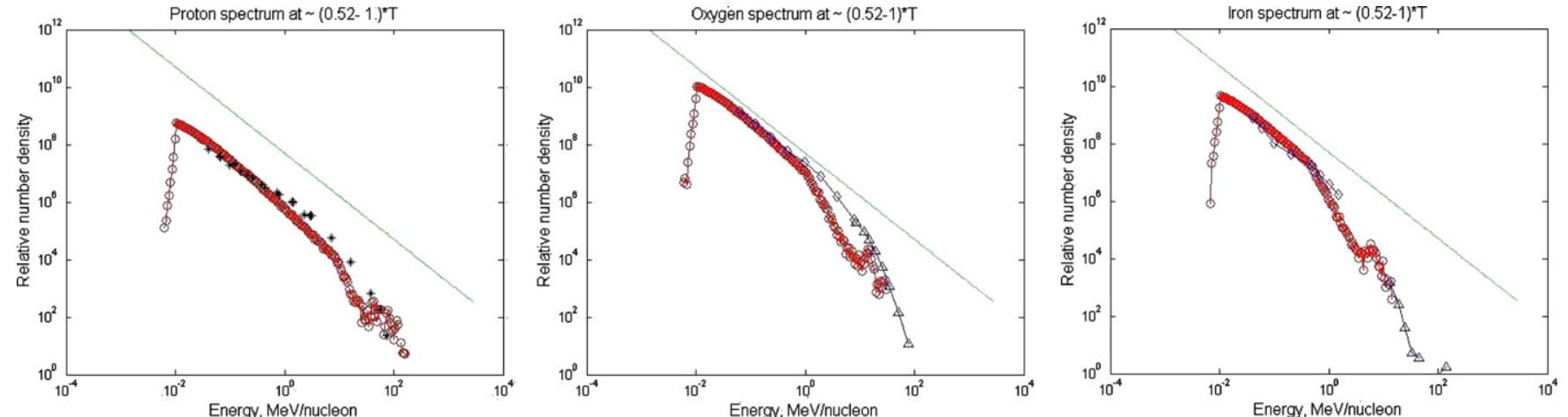

Figure 2. Spectra for (a) protons, (b) oxygen, and (c) iron ions at $1 \mathrm{AU}$ integrated over a $24 \mathrm{hr}$ time interval prior to the shock-arrival time. Modeling results are shown in red. EPAM and EPS measurements are shown by stars (in panel a). ULEIS and SIS measurements integrated over the same time interval are shown by blue diamonds and triangles (in panels b and c), respectively. See text for details. The straight line shows the theoretical limit for an instantaneous power-law spectrum corresponding to the shock-compression ratio $\sim 2.5$ at $1 \mathrm{AU}$.

(A color version of this figure is available in the online journal.)

drawn to guide the eye. Note that this is not a theoretical limit for the event-integrated spectra.

Corresponding $A C E$ measurements for iron and oxygen ions integrated over $\sim 24 \mathrm{hr}$ prior to the shock arrival are also plotted. We use ULEIS (Mason et al. 1998) and SIS (Stone et al. 1998) particle detectors to estimate integrated fluxes and spectra. The low-energy part of the spectra are fitted to ULEIS measurements (shown by diamonds) and the high-energy part of the heavy ion spectra are fitted to the SIS measurements (shown by triangles). Solar proton spectra for the 2001 September 29 event were obtained from the EPAM instrument on $A C E$ (Gold et al. 1998), and the energetic particle sensors (EPS) on NOAA's GOES-8 satellite (Onsager et al. 1996). The GOES data are available at http://spidr.ngdc.noaa.gov/spidr/in the form of corrected differential intensities. The fluence spectra were obtained by integrating hourly average intensities over the $24 \mathrm{hr}$ preceding the arrival of the shock. The EPAM measurements were made with two essentially identical telescopes (LEMS30 and LEMS120), which view, on average, at $30^{\circ}$ and $120^{\circ}$ to the Earth-Sun line. To obtain spectra in the rest frame of the solar wind, we corrected the energy intervals and intensities for the Compton-Getting effect using an average solar wind speed of $513 \mathrm{~km} \mathrm{~s}^{-1}$ as measured by the ACE/SWEPAM instrument.

The modeled fluxes are plotted in arbitrary units and are scaled to the observed flux magnitudes in the figure. For every species (i.e., proton, oxygen, and iron ions), we use the same scaling factor for all energy channels. This point needs some clarification. As discussed above, from the shock parameters and the local interplanetary environment, we determine the excited wave spectrum based on the quasi-linear formulation by Gordon et al. (1999). This determines the diffusion coefficient for a quasi-parallel shock. From the diffusion coefficient, we obtain the local maximum energy and spectra for protons and heavy ions at the shock front. In this process, the shape of the instantaneous power-law spectra at the shock is fully determined by Equation (1). Ahead of the shock at $1 \mathrm{AU}$, the observed spectra are the result of a combination of many individual instantaneous spectra as "sources" from earlier times. These "sources" provide particles that propagate to $1 \mathrm{AU}$, subject to pitch angle scattering and magnetic focusing. Thus, the resultant observed spectra are not single-power law ones and the maximum particle energy is defined by $p_{\max }$ at earlier times. The transport process is certainly energy dependent and the observed spectra at $1 \mathrm{AU}$ are thus complicated. In our model, apart from one scaling parameter that is used to scale with the observed flux at one energy and a particular time, no other fitting or normalizations are made at all. Everything is computed $\mathrm{ab}$ initio. This one scaling parameter is physically related to the injection efficiency and escape efficiency. Since both parameters are largely unknown and are likely to vary from event to event, we treat this scaling parameter as a free parameter and make no attempt in this work to obtain the flux or spectra in a physical unit. In future work, we plan to perform a statistical study of many SEP events and examine how this overall multiplicative constant may vary from event to event.

Figure 2 presents reasonably good agreement in the shape of the modeled spectra and the $A C E$ and GOES-8 measurements. Since the integrated spectra result from overlaying a series of instantaneous spectra at different times, the cumulative spectrum reflects weakening of the shock with time. The maximum energy to which a particle can be accelerated decreases as the shock propagates and the background magnetic field decreases, and only those high-energy particles that were trapped at an earlier time and then subsequently leaked out contribute to the spectrum, resulting in a broken power-law feature with the clear break in energy ordered by $\sim(Q / A)^{2}$. This trend is reflected in observations by SIS of iron and oxygen ions in the high-energy range up to $100 \mathrm{MeV} /$ nucleon.

We need to explain this briefly. The maximum energy to which a shock can accelerate particles at any given moment depends on location (see Figure 1). The maximum momentum $p_{\max }^{H}$ of heavy ions is related to the maximum momentum $p_{\max }^{p}$ of protons through the minimum $k$ satisfying a local resonance condition $p_{\max }^{H}=(Q / A) p_{\max }^{p}$. As the shock propagates, it slows down. Furthermore, the magnitude of both the ambient and turbulent magnetic field decreases (with the former between $r^{-3}$ and $r^{-4}$ and the latter $r^{-3}$ in a WKB approximation). These factors combine to yield maximum energies to which particles of different species can be accelerated, decreasing with increasing heliocentric distance (Zank et al. 2000). Due to these effects, the maximum energy of the escaping particles decreases and the high-energy part of the spectrum starts to "break." Because of the $(Q / A)^{2}$ dependence on energy, the break point of the spectra for different ion species will be ordered by the same factor.

Figure 3 presents modeled fluxes of iron and oxygen ions at $1 \mathrm{AU}$ for four representative energies from the moment the shock was launched until its arrival at $1 \mathrm{AU}$. The most energetic ions of $10 \mathrm{MeV} /$ nucleon contribute to the overall spectrum within the first 5-15 hr. After that, their input is negligible. Low-energy particles of 200 and $570 \mathrm{keV} /$ nucleon are still accelerated as 

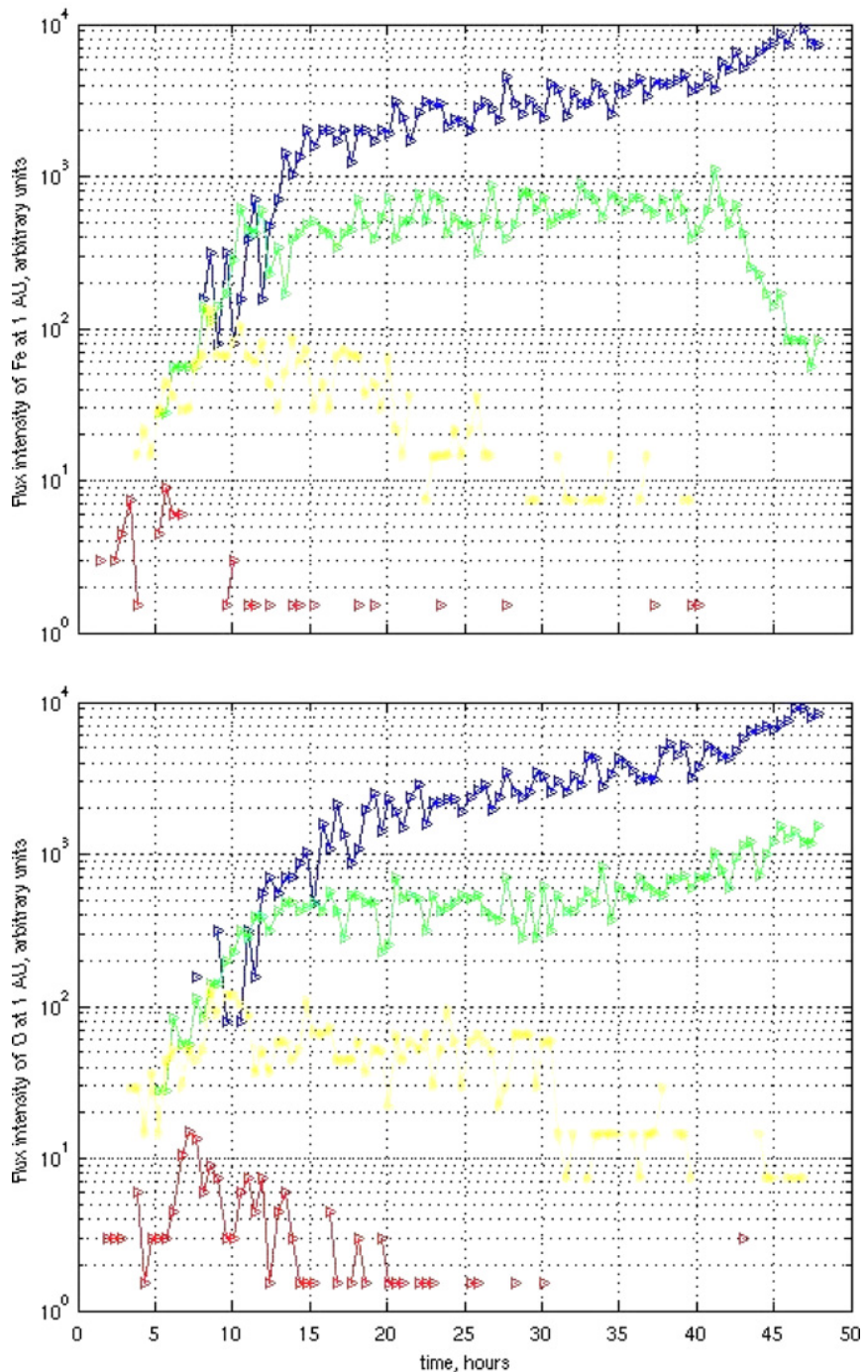

Figure 3. Time-intensity profiles (in arbitrary units) of iron ions (a) and oxygen ions (b) obtained with the PATH model. Representative energies are (from top to bottom): $0.2,0.57,2$, and $10 \mathrm{MeV} /$ nucleon. Time is in hours starting from the shock launch at $0.1 \mathrm{AU}$ until the shock arrival at $1 \mathrm{AU}$.

(A color version of this figure is available in the online journal.)

the shock propagates (see Figure 1 for the maximum energy of accelerated particles), and some escape from the evolving shock. They continuously contribute to the integrated spectrum. A portion of the low-energy particle distribution is trapped behind the shock and has a major impact on the spectrum at the shock-arrival time.

\section{DISCUSSION}

We have modeled the gradual SEP event of 2001 September 29 , and our results show good agreement with particle spectra observed by $A C E$ and $G O E S-8$ at $1 \mathrm{AU}$. Modeled particle fluxes at $1 \mathrm{AU}$ in the energy range below $10 \mathrm{MeV} /$ nucleon show the expected physical evolution in time. In this section, we address several issues. One is the constraint of using a quasiparallel shock to model a real SEP event. This implies that the shock connection to the spacecraft by the interplanetary magnetic field remains in a quasi-parallel configuration as it propagates from the solar corona to $1 \mathrm{AU}$. If acceleration by the 2001 September 29, shock event was dominated by quasiperpendicular diffusion, the maximum energy would have a

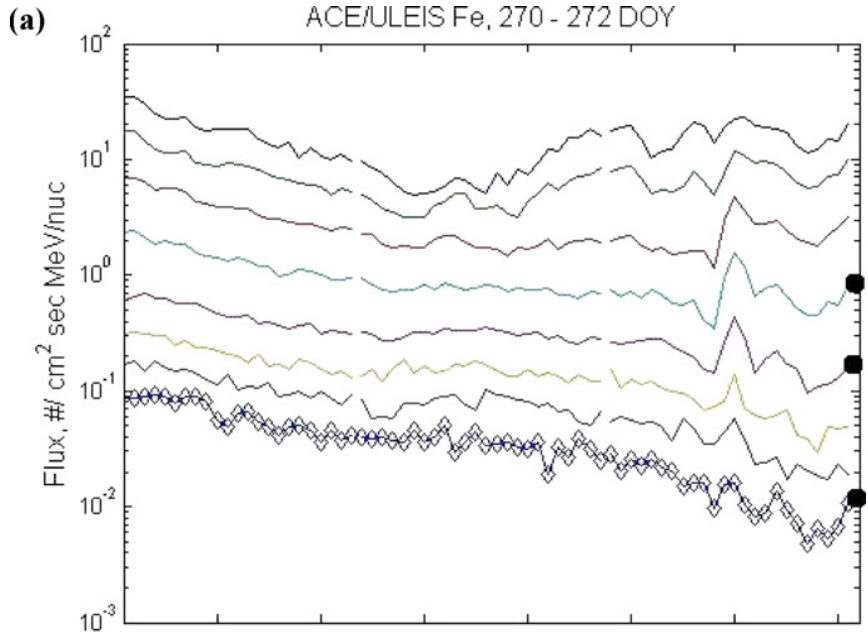

(b) $10^{2} \quad$ ACE/ULEIS 0. 270-272 DOY

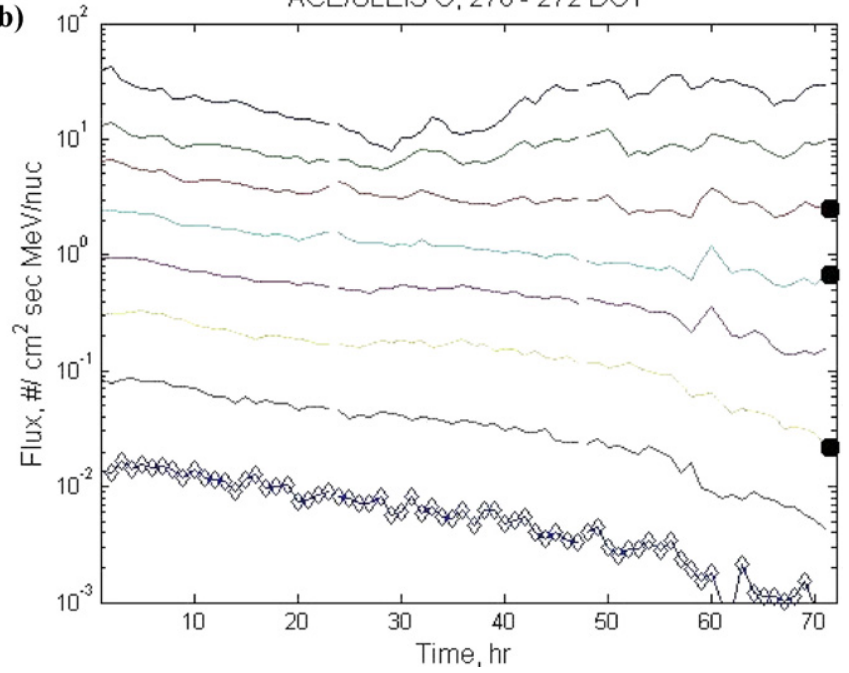

Figure 4. Time-intensity profiles of iron ions (a) in eight energy ranges (from top to bottom): $0.035-0.04,0.04-0.08,0.08-0.16,0.16-0.32,0.32-0.64,0.64$ $0.905,0.905-1.28,1.28-1.81$, in $\mathrm{MeV} /$ nucleon detected by ULEIS. Timeintensity profiles of oxygen ions (b) are shown in the following energy ranges (from top to bottom): 0.04-0.09, 0.09-0.16, 0.16-0.32, 0.32-0.64, 0.64-1.28, $1.28-2.56,2.56-5.12,5.12-10.4$, in $\mathrm{MeV} /$ nucleon. Time is in hours starting from 2001 September 27. Note the enhanced background due to the previous strong SEP event of 2001 September 24-25. Contribution from the modeled event starts to dominate low-energy fluxes at $\sim 28 \mathrm{hr}$. The arrival of the shock at $1 \mathrm{AU}$ corresponds to $57 \mathrm{hr}$ approximately.

(A color version of this figure is available in the online journal.)

different dependence on $(Q / A)$, and our ability to fit the data would be impossible (Zank et al. 2006, 2007). The fact that our results agree with observations justifies our assumption of a quasi-parallel shock for this event. In general, of course, it is better to model an event using a two-dimensional code. This work is currently in progress.

We now examine the validity of our selection criteria (2), i.e., there is a local ESP phase when the shock passes $1 \mathrm{AU}$. Figure 4 shows time-intensity profiles for (a) iron ions and (b) oxygen ions derived from ULEIS measurements starting from September 27. One can identify a clear local maximum of ion fluxes in eight energy channels around the shock passage at $t \sim 60 \mathrm{hr}(t=0$ corresponds UT 00:00 of September 27). Before $t \sim 60 \mathrm{hr}$, the time-intensity profile is an interplay of the current shock and the earlier shock. If only the earlier shock were present, then we would expect to see an exponential decay in the time-intensity profile. This is indeed the case for 
the first $\sim 20 \mathrm{hr}$. If we extrapolate particle fluxes from the first event to later times, the corresponding flux level would be several orders of magnitude smaller (in the energy range of $\leqslant 200 \mathrm{keV}$ ), and several times smaller for higher energies due to this decrease than that of the second (modeled) event at the same times. Starting from $t \sim 20$ to $30 \mathrm{hr}$, however, the time-intensity profiles (for all ULEIS energy channels) show a clear departure from the exponential decay. These enhancements in the intensity profiles also exhibit clear energy dispersion, appearing first at high energies and then at lower energies. These are the signatures of particle acceleration at the second shock. These particles are trapped in the downstream region of the first shock and can be reprocessed by the second shock. We note here a very important concept of "reprocessing" (Li \& Zank 2005). The presence of the first shock may set up enhanced turbulence and an additional seed population for the acceleration process at the second shock. This scenario can naturally explain the observations by Gopalswamy et al. (2004), which have shown that large SEP events are likely associated with multiple CME-driven shocks. However, since the preceding shock is very strong in this particular event, there are high-energy particles with energies that exceed the maximum achievable energy for the second shock. A portion of these particles (depending on their pitch angles) can pass through the second shock with little or no further acceleration because of the absence of resonant waves. Consequently, their time-intensity profiles show no local maxima. Of course, the presence of the second shock will still affect their propagation and will lead to a more sophisticated time-intensity profile. This scenario agrees with SIS measurements for iron and oxygen ions (see Figure 5). One can notice somewhat enhanced fluxes in the lower energy range (below $\sim 10 \mathrm{MeV}$ ), presumably due to particle re-acceleration at the second shock. There is no noticeable additional acceleration at the higher energy end of particle spectra. At lower energies (as seen from Figure 5), particles will be reprocessed by the second shock, because the injection energy is expected to be smaller than that of the preceding shock (due to a stronger turbulence level). We expect a relatively high flux of low-energy particles to be accelerated at the second shock, creating a local maximum in the low-energy range of the time-intensity profile and resulting in a spectrum defined by the second-shock parameters. The resultant spectrum at $1 \mathrm{AU}$ will have a rollover. This feature can be seen in Figure 2 for all three particle species. The overlap of the ULEIS and SIS measurements in the range from 5 to $10 \mathrm{MeV} /$ nucleon for oxygen ions clearly reveals the spectral "break." Similar behavior for the heavy ion spectra was discussed by Cohen et al. (2005) for a sequence of strong gradual SEP events during 2003 October-November.

For the reasons discussed above, we believe that for low energies, the contribution from the previous shock is small, at least for about $30 \mathrm{hr}$ prior to arrival of the second (modeled) shock. Indeed, the background flux is at least from several times to an order of magnitude smaller (depending on energy range) than the accelerated particle flux. We did not subtract a pre-event background from our particle spectra in Figure 2. For highenergy particles, we assume the second shock can reprocess particles accelerated at the first shock up to the maximum achievable energy at the second shock by assuming $\theta=0$. This, of course, is only a coarse approximation. In a way, we assign all the effects of the first shock to an efficient acceleration at the second shock so that it can be treated as a parallel shock case (with the maximum energy evaluated by $\theta=0$ ). How well
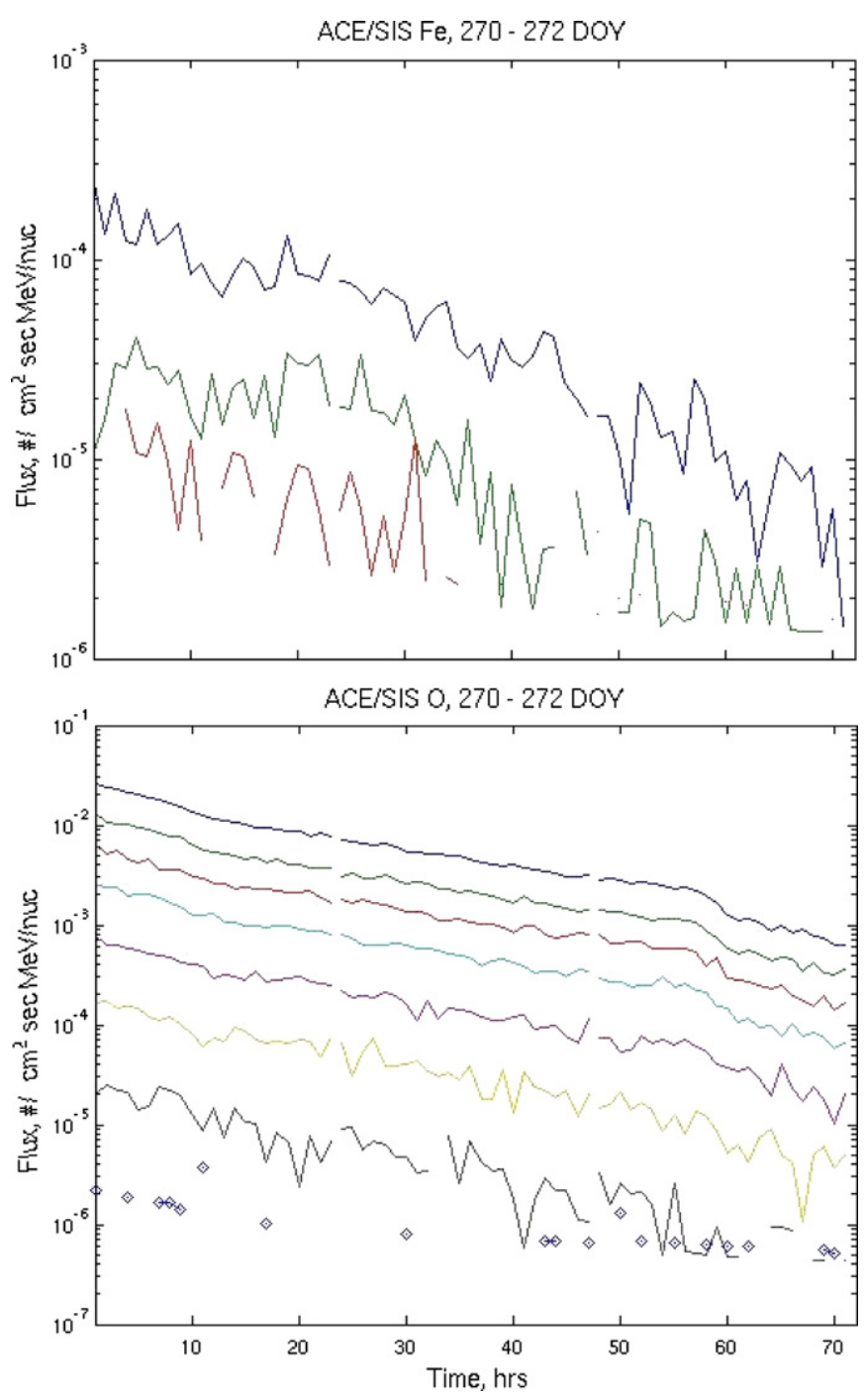

Figure 5. Time-intensity profiles of iron ions (a) for the following representative energies (from top to bottom): 13.3, 19.3, 25.1, in MeV/nucleon detected by SIS. Time-intensity profiles of oxygen ions (b) are shown for the following representative energies (from top to bottom): 8.7, 11.9, 15.0, 19.0, 25.7, 34.8, $50.8,76.2$, in $\mathrm{MeV} /$ nucleon detected by SIS. Time is in hours starting from 2001 September 27.

(A color version of this figure is available in the online journal.)

this assumption holds can only be examined by comparing with observations. Three energy channels for iron and oxygen ions $(0.2,0.57$, and $2 \mathrm{MeV} /$ nucleon) in Figure 3 correspond roughly to the energy channels in Figure 4 shown by black dots on the right axes. Modeled fluxes in the low-energy range show an increase within 10-15 hr after the shock was launched (Figures $3(\mathrm{a})$ and (b)). This timing approximately corresponds to $25 \mathrm{hr}$ in Figures 4(a) and (b). Observations show that low-energy fluxes start to increase at about the same time. The shock-arrival time is indicated by several peaks around $57 \mathrm{hr}$ in Figures 4(a) and (b). Corresponding modeled fluxes show local maxima in lowenergy channels and decreases in high-energy channels. These results qualitatively agree with observations and capture the main features of flux evolution at $1 \mathrm{AU}$ during a major $\mathrm{SEP} /$ ESP event.

We note here, however, that to better understand many SEP events, especially large SEP events, it is necessary to construct a detailed model that treats particle acceleration at a sequence of CME-driven shocks. A typical CME has a 
complex structure (Smith et al. 2001; Tsurutani et al. 2003; Skoug et al. 2004), and an associated SEP event may be the result of two or more interacting shocks. Furthermore, multiple CMEs are often responsible for large gradual SEP events. The $A C E$ List of Disturbances and Transients identifies a number of distinct multiple shock occurrences during intervals of 2-4 days. As discussed earlier, the leading shock(s) can yield an enhanced interplanetary turbulence level as well as enhanced seed population for a subsequent shock; therefore, the observed time intensity and dynamic particle spectra between two shocks at $1 \mathrm{AU}$ are an interplay of both shocks and separating effects from these two close shocks is hard if not impossible. Ideally, one shall first model both shocks and compute the turbulence level upstream of the first shock as well as its transmission to the downstream and propagation to the second shock; then determine the acceleration process at the second shock; and follow the transport of energetic particles in the presence of both shocks.

In summary, we have shown that there is a good agreement between our model calculations and the observed spectra for the specific event. That the PATH model can describe fluxes and spectra of the low-energy particles for the 2001 September 29 event well suggests that we have identified the basic physics describing particle acceleration at CME-driven shocks. However, a pre-event background needs to be taken into account to model fluxes at the high-energy end. On the basis of the analysis presented here, we propose that our approach can model an isolated SEP event. With subsequent modifications, our approach is applicable to a complex event with pre-accelerated particles from a previous strong gradual SEP event or multiple CMEdriven shocks. In this case, the PATH model needs to be tuned to specific solar wind conditions and a pre-event background (by modification of particle injection into the shock). Currently, the PATH model applies to a quasi-parallel shock only. We are working on extending the model to two-dimensional geometry and including perpendicular diffusion at an oblique shock. We are also planning to incorporate contributions from flare particles. Our first results show that physics-based models may play an important role in understanding space weather.

The authors acknowledge the partial support of NASA grants NNG04GF83G, NNG05GH38G, NNG05GM62G, a Cluster University of Delaware subcontract BART372159/ SG, and NSF grants ATM0317509, and ATM0428880. We thank the $A C E$ ULEIS, SIS, EPAM, and SWEPAM teams and the $A C E$ Science Center for providing the $A C E$ data. We are also grateful for the availability of GOES-8 data from NOAA at http://spidr.ngdc.noaa.gov/spidr/. The work at Caltech was supported by NASA under grants NAG5-12929 and NNX06AC21G. We would like to thank the referee for valuable comments.

\section{REFERENCES}

Cohen, C. M. S., et al. 2005, J. Geophys. Res., 110, (A09S16), doi: 10.1029/2005JA011004

Desai, M. I., Mason, G. M., Gold, R. E., Krimigis, S. M., Cohen, C. M. S., Mewaldt, R. A., Mazur, L. E., \& Dwyer, J. R. 2006, ApJ, 649, 470

Ellison, D. C., \& Ramaty, R. 1985, ApJ, 298, 400

Galinsky, V. L., \& Schevchenko, V. I. 2007, ApJ, 669, L109

Gold, R. E., et al. 1998, Space Sci. Rev., 86, 541

Gopalswamy, N., Yashiro, S., Lara, A., Kaiser, M. L., Thompson, B. J., Gallagher, P. T., \& Howard, R. A. 2003, Geophys. Res. Lett., 30, doi:10.1029/2002GL016435

Gopalswamy, N., et al. 2004, J. Geophys. Res., 109, A12015

Gordon, B. E., Lee, M. A., Mobius, E., \& Trattner, K. J. 1999, J. Geophys. Res., 104 (A12), 28263

Kahler, S. W., Sheeley, N. R., Howard, R. A., Koomen, M. J., Michels, D. J., McGuire, R. E., Von Rosenvinge, T. T., \& Reames, D. V. 1984, J. Geophys. Res., 89, 9683

Kallenrode, M.-B. 2003, J. Phys. G: Nucl. Part. Phys., 29, 965

Lee, M. A. 1983, J. Geophys. Res., 88, 6109

Lee, M. A. 2005, ApJ, 158, 38

Li, G., \& Zank, G. P. 2005, 29th Inter. Cosmic Ray Conf. (Pune: ICRC), 1, 173

Li, G., Zank, G. P., \& Rice, W. K. M. 2003, J. Geophys. Res., 108 (A2), 1082, doi: 10.1029/2002JA009666

Li, G., Zank, G. P., \& Rice, W. K. M. 2005, J. Geophys. Res., 110 (A06104), doi: 10.1029/2004JA010600

Malkov, M. A., \& Drury, L. O’C. 2001, Rep. Prog. Phys., 64, 429

Mason, G. M., et al. 1998, Space Sci. Rev., 86, 1-4, 409

Ng, C. K. 2007, 6th AIP Conf. Proc., Turbulence and Nonlinear Processes in Astrophysical Plasmas, ed. D. Shaikh \& G. P. Zank (Melville, NY: AIP), CP932, 271

Onsager, T. G., et al. 1996, SPIE Conf. Proc. 2812 Operational Uses of the GOES Energetic Particle Detectors, in GOES-8 and Beyond, ed. E. R. Washwell (Bellingham, WA: SPIE), 281

Rice, W. K. M., \& Zank, G. P. 2003, Adv. Space Res., 31, 901

Rice, W. K. M., Zank, G. P., \& Li, G. 2003, J. Geophys. Res., 108(A10), 1369, doi: 10.1029/2002JA009756

Skoug, R. M., Gosling, J. T., Steinberg, J. T., McComas, D. J., Smith, C. W., Ness, N. F., Hu, Q., \& Burlaga, L. F. 2004, J. Geophys. Res., 109, A09102, doi:10.1029/2004JA010494

Smith, C. W., et al. 2001, Solar Phys., 204, 229

Stone, E. C., et al. 1998, Space Sci. Rev., 86, 357

Tsurutani, B. T., Wu, S. T., Zhang, T. X., \& Dryer, M. 2003, Astron., \& Astrophys., 412, 293

Tylka, A. J., Cohen, C. M. S., Dietrich, W. F., Lee, M. A., Maclennan, C. G., Mewaldt, R. A., Ng, C. K., \& Reames, D. V. 2005, ApJ, 625, 474

Vainio, R., \& Laitinen, T. 2007, ApJ, 658, 622

Zank, G. P., Rice, W. K. M., \& Wu, C. C. 2000, J. Geophys. Res., 105(A11), 25079

Zank, G. P., Li, G., Florinski, V., Matthaeus, W. H., Hu, Q., Lario, D., \& Smith, C. W. 2006, J. Geophys. Res., 1 (A6), A06108

Zank, G. P., Li, G., \& Verkhoglyadova, O. P. 2007, Space Sci. Rev., 130, 255

Zank, G. P., Webb, G. M., \& Donohue, D. J. 1993, ApJ, 406, 67 